\title{
Las infecciones nosocomiales y la calidad de la atención médica
}

\author{
Susana N avarrete-N avarro, M.C., ${ }^{(1)}$ M. Sigfrido Rangel-Frausto, M.C., M. en C. ${ }^{(2)}$
}

\section{Navarrete-Navarro S, Rangel-Frausto MS. Las infecciones nosocomiales y la calidad de la atención médica. Salud Publica Mex 1999;41 suppl 1:S64-S68.}

\section{Resumen}

El objetivo más importante de un programa de control de calidad en hospitales es reducir el riesgo de adquirir una infección, la morbilidad y los costos asociados. Es indispensable la organización de un equipo con liderazgo técnico y humanístico que permita: a) identificar los problemas, b) determinar la importancia de cada uno de ellos, c) encontrar sus causas, d) proponer soluciones y e) evaluar continuamente todo el programa. El desarrollo de estas cualidades técnicas y humanísticas por parte del líder y los miembros del programa, así como el uso continuo de las herramientas mencionadas, han producido el único programa validado y altamente efectivo de control de calidad en los hospitales.

Palabras clave: infección hospitalaria; calidad de la atención de salud; México

\author{
Navarrete-N avarro S, Rangel-Frausto MS. \\ The nosocomial infections \\ and the quality of medical care. \\ Salud Publica Mex 1999;41 suppl 1:S64-S68.
}

\section{A bstract}

The main objective of a hospital-acquired infections control program is to decrease the risk of acquisition and the morbidity and costs associated. The organization of a team with technical and humanistic leadership is essential. Every infection control program must also develop strategies that allow: a) identification of the problems, b) to establish the importance of each one, c) to determine their causes, d) to develop solutions and e) the evaluation of the recomended solutions.T he development of technical and humanistic habilities by the leader and the members of the team, and the use of the tools mentioned above have produced the only validate and highly effective program of quality improvement in the hospital.

Key words: cross infection; quality of health care; Mexico
L as infecciones que se adquieren dentro de un hospital son consecuencia del tipo de atención médica que reciben los pacientes y representan un problema de extraordinaria gravedad. Los informes que se publican en todo el mundo muestran que entre 5 y $10 \%$ de los enfermos que se hospitalizan, adquieren por lo menos un episodio de infección durante su estancia en el nosocomio.

Estas infecciones contribuyen a una inadecuada calidad de la atención médica dentro del hospital, ya que la principal expectativa de un enfermo al recibir cuidados médicos es la solución de sus problemas de salud..$^{1-3}$

Los antecedentes de la preocupación por la calidad en la atención médica podrían remontarse al siglo XVII, cuando Sir William Pety se preguntó si la intervención médica era mejor que dejar al paciente sin ayuda alguna o, por otro lado, cuando se evaluaron los desenlaces de la hospitalización de pacientes, durante la guerra de Crimea.

(1) Unidad de Investigación Médica en Epidemiología Hospitalaria, Centro Médico N acional Siglo XXI, Instituto Mexicano del Seguro Social, México.

(2) División de Epidemiología Hospitalaria y Control de Calidad, Instituto N acional de la N utrición Salvador Zubirán, México.

Fecha de recibido: 19 de febrero de 1998 - Fecha de aprobado: 6 de agosto de 1998 Solicitud de sobretiros: Dr. M. Sigfrido Rangel. Edificio C oordinación Regional, 3er. piso. Av. Cuauhtémoc 330 colonia Doctores, 06725 México, D.F. 
Desde el inicio de los años ochenta, en Estados Unidos de América la calidad de la atención médica se ha vuelto una preocupación creciente tanto del público usuario como del sector médico. Así, el continuo escrutinio, el debate y la documentación de la atención médica han disminuido el respeto de la comunidad por los profesionales de la medicina y la enfermería. ${ }^{4}$

Este cambio en la actitud del público hacia las profesiones relacionadas con la salud podría entenderse mejor a partir del marco de las percepciones que Relman ha hecho de la medicina. ${ }^{5}$ Ese autor propone tres grandes revoluciones modernas en la atención médica: la primera fue la expansión (1940-1960); la segunda, la limitación de costos (principios de los ochenta), y la tercera corresponde a la estimación de la responsabilidad (fines de los ochenta).

El periodo de expansión estuvo caracterizado por un rápido aumento en la cantidad de hospitales, de médicos y de avances de la técnica médica. Durante el mismo, el aumento en la proporción del producto nacional bruto que se invirtió en salud se elevó de 4.4 a $12 \%$.

En el segundo periodo, la creciente preocupación por los costos excesivos de la atención médica dio lugar a una serie de medidas que incluyeron la elaboración de categorías diagnósticas, con retribuciones fijas por parte de las compañías de seguros.

Esta etapa condujo a la tercera revolución o estimación del riesgo, como un componente fácil de medir y que permitía la estimación indirecta de la calidad, primer paso para tener un panorama muy general y superficial de lo que era la calidad de la atención médica que reciben los pacientes. ${ }^{5}$

Debido a que han surgido varias confusiones en torno a los conceptos relacionados con calidad de la atención médica, algunos autores han diseñado varias definiciones que marcan las diferencias entre dichos conceptos.

Ruelas $^{6,7}$ define la gestión de la calidad como el conjunto de acciones sistematizadas y continuas, dirigidas a prevenir $\mathrm{y} / \mathrm{o}$ resolver oportunamente problemas o situaciones que impidan otorgar el mayor beneficio posible o que incrementen los riesgos en los pacientes mediante la vigilancia o el seguimiento, la evaluación, el diseño, el desarrollo y los cambios organizacionales. Para lograr la gestión de la calidad se aplican varias estrategias por medio de las cuales se da importancia a los conceptos que integran la gestión.

En relación con lo anterior, en el campo de la atención médica se ha hecho gran énfasis en la vigilancia y en las auditorías o evaluaciones como estrategias para medir la calidad del servicio médico. Dichas estrate- gias se enfocan en los aspectos técnicos del proceso médico dejando de lado el desarrollo de las habilidades humanísticas del equipo de trabajo que brinda el servicio de salud. Quienes las aplican consideran que la calidad se logra detectando los malos elementos y sacándolos del grupo, esforzándose por encontrar mejores herramientas de inspección, con una elevada sensibilidad y especificidad. La fuerza de trabajo atemorizada por la vigilancia tiene que hacer frente a este tipo de estrategias. El miedo generado por el enfoque disciplinario conduce al enajenamiento, la distorsión de la información y la pérdida de la oportunidad de aprendizaje.

Otra estrategia es el mejoramiento continuo, que se caracteriza por fomentar y reconocer la importancia de la participación de los miembros de una organización mediante el trabajo en equipo, estimulando la creatividad, la aplicación de métodos estadísticos y el análisis de los problemas.-8

El mejoramiento continuo se aplica cada día con mayor frecuencia al área de la atención para la salud, y cuenta con el respaldo de teóricos japoneses y norteamericanos como Ishikawa, Deming y Juran..$^{9-11}$ Este concepto resulta del proceso dialéctico de la administración, en el que no se concibe la calidad como una meta fija, sino como un atributo de un servicio, que puede ser mejorado continua y paulatinamente; se basa en el esfuerzo permanente y progresivo, que va dando satisfacción y sensación de logro a los equipos de trabajo, sin causar la impresión de algo difícil o imposible de alcanzar.

Esta estrategia depende de la comprensión y la revisión de los procesos, y tiene como base las observaciones de los trabajadores, la reducción en la duplicación, el desperdicio y la baja calidad dentro del proceso, facilitando y conduciendo la participación del mismo trabajador en la toma de decisiones y la resolución del problema de la calidad.

Cuando el proceso se comprende claramente y es constante en los fines que busca alcanzar, cuando el miedo no controla el ambiente y el aprendizaje se guía por información exacta y sólidas reglas de reducción, y cuando los proveedores de los servicios permanecen en diálogo con sus subalternos, aplicando la voluntad y el talento de todos los trabajadores a la identificación de varias formas de realizar el trabajo, las posibilidades de mejoramiento de la calidad son casi ilimitadas.

Tomando en cuenta lo arriba expuesto, este último enfoque parece ser uno de los más adecuados para un programa de control de infecciones. Es importante mencionar que este enfoque se basa en los siguientes puntos: a) estructura, $b$ ) proceso y c) resultados. 
Dentro de la estructura, el enfoque se dirige hacia: a) el diseño organizacional (a partir del organigrama, de manuales de organización y del funcionamiento del hospital) y b) recursos (humanos, materiales y financieros).

En lo referente a los procesos, éstos estarán centrados en las interacciones humanas y técnicas de: los trabajadores-pacientes, trabajadores-trabajadores y trabajadores-institución.

Por su parte, los resultados estarán dirigidos a la satisfacción humana y técnica de los trabajadores, los pacientes y sus familiares, y la institución.

Tomando en cuenta lo anterior, la aplicación de la estrategia de mejoramiento continuo es útil en el trabajo médico, incluyendo la labor realizada por el grupo o comité que controla y vigila las infecciones de adquisición intrahospitalaria ya que, a final de cuentas, este último debe operar como un verdadero equipo de trabajo.

\section{El control de infecciones como programa de calidad}

Este programa ha sido considerado como uno de los más importantes, en lo referente a control de la calidad hospitalaria; además, es uno de los que ha demostrado tener mayor eficiencia en materia de estudios de investigación clínica. ${ }^{12}$

El objetivo principal de un programa de este tipo es mejorar la eficiencia en el control de las infecciones, disminuyendo su frecuencia y costos de operación, evitando, por lo tanto, gastos innecesarios para la institución que presta el servicio médico pero, sobre todo, contribuyendo al mejoramiento de la calidad en la atención médica. ${ }^{13}$

Debido a lo anterior, la comprobación de que un hospital cuenta con un programa de control de infecciones en operación es fundamental en el proceso de acreditación o certificación de hospitales, recomendado por la Organización Panamericana para la Salud. ${ }^{14}$

\section{El comité para el control de infecciones como equipo de calidad}

Son diversos los términos que se han utilizado para denominar a los equipos que trabajan en los programas de calidad de la atención médica; su variedad depende del enfoque o de la corriente de la calidad existente en el momento. Esos nombres van desde "círculos", "equipos" o "escuadrones de calidad", hasta "equipos de proyecto", entre otros.

Los equipos de calidad son grupos pequeños (de 4 a 20 personas) que se reúnen voluntaria y periódi- camente (cada semana o dos), para detectar, analizar y buscar soluciones a los problemas que se suscitan en su área de trabajo y que repercuten en la obtención de una mejoría continua de la calidad dentro del hospital.

Las ideas básicas de los equipos de calidad son: servicio voluntario, autodesarrollo, desarrollo mutuo, participación de todos los miembros y continuidad. Además, uno de sus objetivos es la creación de una conciencia de calidad (cultura de calidad) a partir del trabajo en equipo, participando activamente en la resolución de los problemas que, en este caso, son los relacionados con las infecciones que se presentan en el hospital. Los equipos de calidad dirigen su atención al desarrollo de los recursos humanos mediante la motivación y la participación en la resolución de problemas concretos, y su filosofía se fundamenta en las teorías de Argyris, Mc Gregor y Herzberg. ${ }^{15-17}$

\section{Liderazgo de excelencia}

El responsable del comité o equipo para el control de infecciones deberá ser un verdadero líder de excelencia, lo cual facilitará la dinámica de trabajo del equipo. Para eso se requiere, en primer lugar, que el líder y los miembros del equipo desarrollen habilidades tanto técnicas como humanísticas, además de utilizar herramientas para la previsión, la planeación, la ejecución y el control del trabajo médico, que en este caso está directamente relacionado con las infecciones nosocomiales.

Entre las habilidades técnicas se encuentran el manejo del proceso administrativo, administración por objetivos, organización del tiempo, toma de decisiones, manejo efectivo de reuniones, hablar en público, delegación de autoridad y manejo del estrés.

En relación con las habilidades humanísticas se pueden mencionar la motivación, la comunicación asertiva, el análisis de transacciones humanas y el manejo del enojo y conflicto, entre otras.

\section{Beneficios del trabajo en equipo en el comité para el control de infecciones}

\section{Para la institución}

- Proporcionar satisfacción y contribuir a la realización del trabajador, mejorando la calidad de vida laboral en el hospital.

- Enriquecimiento horizontal del trabajo (cada trabajador se convierte en un administrador).

- Incremento de las habilidades de los supervisores para dirigir su servicio o departamento, ya 
que se facilita la comunicación entre el(la) supervisor(a) y sus subordinados, lo cual integra mejor al personal.

- Conciliación de los intereses comunes del personal y de la institución, creando una conciencia de calidad del trabajo entre los recursos humanos que laboren en el hospital.

- Aprovechamiento de la energía y la creatividad del personal.

- Mejoramiento de la efectividad organizacional.

- Mejores relaciones entre el personal de base y el de confianza, lo cual aumenta la disponibilidad del mismo prestador de servicios.

\section{Para el trabajador}

- Aumento de la capacidad para resolver problemas sobre infecciones.

- Mayor participación en la solución de esos problemas, mismos que afectan directa o indirectamente al trabajador.

- Oportunidad de aprender más.

- Ser escuchados por el personal de la Dirección.

- Obtener reconocimientos o estímulos por su trabajo.

- Posibilidad de mejorar sus sistemas de trabajo y las condiciones del mismo.

\section{Actividades de identificación y solución de problemas de infecciones}

Una vez iniciados la sensibilización y el desarrollo de habilidades humanísticas del equipo de trabajo, se realizan las actividades correspondientes a cada una de las etapas que forman el rehilete de mejoría continua de la calidad, de acuerdo con el enfoque propuesto por Palencia. ${ }^{18, *}$ En el cuadro I se muestran los pasos que se siguen a este respecto.

Los cuatro pasos que debe contemplar el rehilete del programa de control de infecciones con un enfoque hacia la mejoría de la calidad son: a) identificar cuáles son los problemas relacionados con el control de infecciones; $b$ ) determinar cuál es el peso o importancia de cada uno de los problemas identificados; c) reconocer las causas de los problemas, y d) proponer

\footnotetext{
* Palencia AM. Cómo diseñar un programa de mejoría continua de calidad como instrumento para la planeación y el desarrollo de actividades encaminadas a mejorar la calidad del servicio en un hospital. Diplomado en Mejoría Continua de la Calidad, 1995. Comunicación personal.
}

\section{Cuadro I \\ Dinámica de trabajo del EQUIPO de CALIDAd EN CONTROL DE INFECCIONES}

1. Los miembros del equipo o comité de control de infecciones enlistan los problemas respecto a infecciones nosocomiales.

2. Por votación seleccionan el problema número 1.

3. Se analiza el problema y se recolecta información sobre el mismo a fin de detectar sus causas.

4. Se estudian las causas y se busca y propone la mejor solución.

5. Se presenta a la directiva el caso (problema), junto con sus alternativas de solución.

6. Se instituye el cambio y se notifican los resultados.

alternativas de solución dirigidas al problema de mayor importancia.

- En primer término se utilizan herramientas como la lluvia de ideas, encuestas de opinión, registros estadísticos y relatos de experiencias del mismo equipo.

- Para el segundo paso, que es la jerarquización de los problemas, se utilizan la técnica de magnitud, trascendencia, vulnerabilidad y factibilidad (MTVF), el diagrama de Pareto, la votación grupal y la técnica de grupo nominal.

- Para identificar las causas del problema se recurre a los diagramas de Ishikawa, de flujo y hojas de inspección.

- En la etapa de propuesta de alternativas de solución al problema se utilizan herramientas como: formatos de acuerdos, manuales de organización, diseño de indicadores y diagramas de control entre otros.

En el cuadro I se mencionan, de forma general, los pasos que puede seguir el comité para el control de infecciones.

\section{Indicadores de calidad en infecciones intrahospitalarias}

El trabajo del comité para el control de infecciones, además de aplicar la metodología descrita, debe crear indicadores. ${ }^{*}$ Un indicador se define como el índice o reflejo de una situación dada; es una medida de des-

\footnotetext{
* Palencia AM. Cómo diseñar un programa de mejoría continua de calidad como instrumento para la planeación y el desarrollo de actividades encaminadas a mejorar la calidad del servicio en un hospital. Diplomado en Mejoría Continua de la Calidad, 1995. Comunicación personal.
} 
empeño y una herramienta básica para determinar el comportamiento de una variable. Las principales etapas por las cuales debe pasar un indicador son:

1. Su creación con base en una propuesta y marcando los objetivos del mismo.

2. Su aplicación de acuerdo con la realidad de los servicios médicos, con el objeto de medir su utilidad.

3. Ajuste según sea necesario, tomando como base la experiencia de su utilización en la práctica médica.

Algunos ejemplos de indicadores en infecciones nosocomiales son: tasa de incidencia de infecciones intrahospitalarias; tasas específicas de acuerdo con el tipo de infección (p.e., tasa de infección asociada a catéter, cirugía o sondas, etc.); mortalidad asociada a infecciones nosocomiales, y estancia hospitalaria secundaria a infección, costo-efectividad, costo-beneficio y costo-oportunidad, todas vinculadas a ese tipo de infecciones.

\section{Referencias}

1. D onabedian A. La calidad de la atención médica. D efinición y métodos de evaluación. México, D.F.: Prensa Médica Mexicana, 1991:1-194.

2. Ruiz CHA, Martínez NG, Calva RJM. Bases para la evaluación de la calidad de la atención en las unidades médicas del sector salud. Salud Publica Mex 1990;32:156-169.

3. Haley RW, W hite JW, Culver DH, Hughes JM. The financial incentive for hospitals to prevent nosocomial infections under prospective payment system. A determination from a nationally representative sample. JAMA 1987;257:1611-1614.

4.W enzel RP. Hospital epidemiology. Beyond infection control and toward quality assurance. Clin Microbiol N ewsletter 1998;10:60-62.

5. Relman AS. Reforming the health care system. N Engl J Med 1990;323: 991-992.

6. Ruelas BE. Hacia una estrategia de garantía de calidad. D e los conceptos a las acciones. Salud Publica Mex 1992;34:29-45.

7. Ruelas BE.Transiciones indispensables: de la cantidad a la calidad y de la evaluación a la garantía. Salud Publica Mex 1990;32:108-109.

8. Ruelas BE. Círculos de calidad como estrategia de un programa de garantía de calidad. Salud Publica Mex 1990;32:207-220.

9. Ishikawa K.W hat is total quality control?T The Japanese way. Englewoods Cliff (N Y): Prentice Hall, 1985.

10. Deming W E. Calidad, productividad y competitividad. La salida de la crisis. Madrid: Díaz de Santos,1989.

11. Juran JM, Gryna FM, eds. Manual de control de calidad. Barcelona: Reverté, 1990

12. Haley RW, Culver DH,W hite JW.The efficacy of infection surveillance and control programs in preventing nosocomial infections in US hospitals. Am J Epidemiol 1985;121:182-205.

13. Haley RW, W hite JW, Morgan W M, EmoryTG .The nationwide nosocomial infection rate: A new need for vital statistics. Am J Epidemiol 1985: 121:159-169.

14. Paganini JM, Moraes N H.Acreditación de hospitales para A mérica Latina y el Caribe. Washington, D.C.: 0 rganización Panamericana para la Salud, 1992

15.Argyris ChA.A integrãção individuo-organizãção. São Paulo:Atlas, 1975. 16. MCG regor D M.An uneasy look at performance appraisal. Boston: $\mathrm{H}$ arvard Business Review, 1957.

17. Herzberg F.W ork and nature of man. Cleveland:World Publishing, 1966.

18. PalenciaAM. Cómo desarrollar una cultura de calidad y excelencia en los servicios de salud: apuntes para la docencia.J Health Adm Educ 1993; 11:125-134. 